PART II

DIFFUSE FEATURES 


\title{
A SEARCH FOR DIFFUSE INTERSTELLAR FEATURES IN STARS WITH CIRCUMSTELLAR DUST SHELLS
}

\author{
THEODORE P. SNOW, JR. and GEORGE WALLERSTEIN \\ Astronomy Dept., University of Washington, Seattle, Wash. 98195, U.S.A.
}

\begin{abstract}
We have searched for diffuse features at $\lambda 4430,5780$ and 6613 in the spectra of 17 stars which show infra-red excesses or other grounds for believing that they have dusty envelopes. In no case could we identify diffuse bands that may be ascribed to the circumstellar envelope. We conclude that the grains in circumstellar envelopes are not identical with the grains in the general interstellar medium.
\end{abstract}

\section{Introduction}

Although the diffuse interstellar absorption bands have been studied for over $35 \mathrm{yr}$ no certain identification of the source of the absorption has been made. The general correlation between interstellar reddening and the strength of the diffuse feature at $\lambda 4430$ (Duke, 1951) has been used to associate the diffuse absorption features with interstellar dust. Furthermore, the width of the bands indicate that they are not due to the absorption by discrete transitions of atoms or molecules in the gaseous state; more likely they are caused by a substance that is adsorbed on the grains.

While the increase in band strength with distance indicates that the diffuse bands are in most cases interstellar, rather than circumstellar; the possibility is not ruled out that stars with a sufficient concentration of circumstellar dust should show anomalously strong diffuse bands. Recent observations in the infra-red have shown that many stars have an excess of infrared radiation that can be explained most readily by the hypothesis that circumstellar dust is absorbing radiation from the photosphere and re-radiating it in the infrared. The question naturally arises as to whether the circumstellar dust also results in absorption in the diffuse bands. We have investigated the possibility that the diffuse bands may be present in stars with circumstellar extinction by examining the spectra of stars with infrared excesses or other grounds for suspicion that circumstellar dust may be present. We have investigated stars which we believe to be evolved objects rather than contracting stars*; but can never be certain of the evolutionary state of each object.

\section{Spectroscopic Observations}

We have searched for the diffuse bands at $\lambda 4430,5780,5796$ and 6613 on spectra loaned from the Hale Observatory plate collection as well as some taken at the Hale Observatories in connection with other investigations of infrared stars by one of us

* Except for AB Aur (Herbig, 1960). 
(G.W.). In stars later than about type F0 the blue region of the spectrum is so crowded with lines that the recognition of the $\lambda \mathbf{4 4 3 0}$ band becomes nearly impossible, so we have searched for the features in the visual and red in the cooler stars. Microdensitometer tracings were prepared of the blue spectra, since the $\mathbf{4 4 3 0}$ feature is too broad to identify readily by visual inspection. Continuum points $50 \AA$ on either side of $4430 \AA$ were used to establish a baseline against which the absorption in the band could be measured. The red spectra were examined with a visual projection measuring engine, to distinguish the $\lambda 5780$ and 6613 features from stellar lines.

\section{Photometric Data}

Since we are comparing the correlation of diffuse band strength with visual absorption in the circumstellar environment and the interstellar environment, we must find the absorption due to circumstellar material for each star. Published photometric data were used to estimate the visual absorption in stars with infra-red excesses. The absolute energy distribution was plotted for each star and the emergent stellar flux was separated from the infra-red excess. We can fairly easily establish the total reradiated energy this way except when the infra-red flux is still rising at the longest observed wavelength. In that case we have assumed a characteristic temperature and integrated under the black body distribution at that temperature from the longest observed wavelength to infinity.

While the total absorbed flux may be set equal to the reradiated infra-red flux, the energy distribution of the absorption is not easily determined. For interstellar particles the albedo in the visual region is about 0.7 (Van de Hulst and de Jong, 1969); i.e. only about $\frac{1}{3}$ of the energy subtracted from the beam is absorbed by the particles. For circumstellar particles there is some evidence that the total extinction curve differs from the interstellar case and that the ratio of extinction to reddening also differs (Hyland et al., 1969; Strom et al., 1972). Hyland et al. found that the extinction curve for VY CMa is flat from $3500 \AA$ to $10000 \AA$ and then decreases rapidly to nearly zero at $3.5 \mu$. However, the absorption could not be absolutely zero in the infra-red, since the circumstellar dust radiates profusely out to at least $20 \mu$. In the second paper cited above the authors found a very large ratio of extinction to reddening indicating that the circumstellar dust absorbs rather nonselectively. In calculating the visual absorption we have assumed that the absorption is equal at all wavelengths. For the purpose of calculating the visual absorption we do not need to know the ratio of absorption to scattering, but will comment on that later.

In view of the difficulties presented by the lack of data in the far infra-red, the ambiguity in determining the cut-off point between emergent stellar flux and reradiated infra-red flux, and the unknown spectral distribution of the absorption in optical wavelengths, we have estimated crude bolometric circumstellar extinctions from

$$
A=-2.5 \log \left(\frac{F_{v}}{F_{\mathrm{bol}}}\right)
$$


where $F_{v}$ is the estimated stellar flux which escapes the dust shell directly without loss, $F_{\text {bol }}$ is the total flux of the star, and $A$ is the extinction in magnitudes. Since the bolometric flux is given by the sum of the emergent stellar flux plus the infrared excess flux, we have

$$
A=-2.5 \log \left(\frac{F_{v}}{F_{v}+F_{\mathrm{IR}}}\right),
$$

where $F_{\mathrm{IR}}$ is the estimated infra-red excess flux. We can also estimate crude bolometric optical depths of the dust shell for optical wavelengths from

$$
\tau=-\ln \left(\frac{F_{v}}{F_{v}+F_{\mathrm{IR}}}\right) .
$$

In the case of VY CMa, where we had enough information to estimate a true visual extinction as well as a bolometric extinction from Equation (2), we found them to be approximately equal.

In some cases the stars under consideration were variables with large amplitudes. For these it was necessary to attempt to match phases by using available light curve data. This was difficult and at best quite inexact, because very little information can be found concerning the IR light curves of variable stars (Lockwood and Wing, 1971; Lockwood [preprint] have done some work on Mira variables). In a few cases satisfactory phase matching was not achieved and we could only place upper limits on the circumstellar extinction.

\section{Results}

Table I lists the stars whose spectra we have examined. After the star name or number we list the region searched and the circumstellar absorption calculated as described above. With the exception of RS Ophiuchi and $\varepsilon$ Aur we could find no stars with diffuse band absorption. At $\lambda 4430$ we can set an upper limit of $5 \%$ absorption. No quantitative limit can be set for the bands in the visual region since most of our stars have atomic or molecular lines near $\lambda 5780$ and 6613 . We can only state that there is no noticeable enhancement or widening of these features.

The only star to show evidence of absorption at $\lambda 4430$ is $\varepsilon$ Aur. It is an eclipsing system with a period of $27 \mathrm{yr}$ and an eclipse duration of about two years. The eclipse lasts too long for the eclipsing object to be a normal star and it has been suggested that a small central object surrounded by a cloud of gas or dust may be responsible for the eclipse. For a recent discussion of $\varepsilon$ Aur see Wilson (1971) which contains many further references. We are concerned here not with the nature of the central object, but with its large surrounding envelope. We find that the $\lambda 4430$ band is of equal strength both in and out of eclipse, showing that the $\lambda \mathbf{4 4 3 0}$ absorption is not formed in the object that is in front during the eclipse. Furthermore, the central depth of the $\lambda 4430$ band in $\varepsilon$ Aur is $11 \%$, which is just about what may be expected from an interstellar reddening of $0.34 \mathrm{mag}$. and its distance of about $1000 \mathrm{pc}$. We found from 
TABLE I

Stars investigated for diffuse bands

\begin{tabular}{llllll}
\hline Object & $\begin{array}{l}\text { Spectral } \\
\text { type }\end{array}$ & $\begin{array}{l}\text { Region } \\
\text { searched }\end{array}$ & $\begin{array}{l}\text { Circumstellar } \\
\text { absorption } \\
\text { (mag.) }\end{array}$ & Reference & Remarks \\
\hline$\varepsilon$ Aur & A8Ia & a b c & 0.0 & 13 & $*$ \\
AB Aur & B9 & a & 0.4 & $7,12,15$ & \\
R Aql & M5-M8e & b c & 0.4 & $4,12,17$ & \\
W Cep & K0 Ia & b c & $\leq 0.05$ & 4,17 & \\
VY CMa & M3-M5 Ib & b c & 2.1 & 11,12 & \\
T CrB & M3III+sdB & a & $\leq 0.05$ & $8,13,15$ & $\checkmark$ \\
RW Cyg & M3 Ia & b c & $\leq 0.30$ & 6,17 & \\
U Her & M6-8e & b c & 0.50 & $2,3,16$ & \\
AC Her & F2-K4Ib & a & 0.20 & 4,18 & \\
89 Her & F2 Ia & a & 0.50 & 5 & \\
17 Lep & B8+M2III & a b c & 0.50 & $1,13,15,17$ & \\
48 Lib & B pec & a & 0.15 & 13,14 & \\
T Mon & F8-K0Ib & a b c & & 6,17 & \\
U Ori & M6-8e & b c & $\leq 0.15$ & 6,17 & \\
W Ser & F5e & a & & 19 & \\
VX Sgr & M4 & b c & 1.0 & $9,10,12$ & \\
SV Vul & F8-K0Ib & a b c & & 4 & \\
\hline
\end{tabular}

$a=4430 \quad * \quad$ at max. extinction at min. is $0.8 \mathrm{mag}$.

$\mathrm{b}=5780+$ not known but presently under study

$c=6613 \quad \nabla$ spectra taken during outburst of 1946

$\triangle$ spectra taken near maximum light.

1. Blanco, V. M., Demers, S., Douglas, G. G., and Fitzgerald, M. P.: 1968, Publ. U.S. Naval Obs. XXI.

2. Dyck, H. M. and Lockwood, G. W.: private communication.

3. Forrest, W.: private communication.

4. Gehrz, R. D. and Woolf, N. J.: 1970, Astrophys. J. Letters 161, L 213.

5. Gillett, F. C., Hyland, A. R., and Stein, W. A.: 1970, Astrophys. J. Letters 162, L21.

6. Gillett, F. C., Merrill, K. M., and Stein, W. A.: 1971, Astrophys. J. 164, 83.

7. Gillett, F. C. and Stein, W. A.: 1971, Astrophys. J. 164, 77.

8. Hofleit, D.: 1964, Yale Catalogue of Bright Stars.

9. Humphreys, R. M. and Lockwood, G. W.: 1972, Astrophys. J. Letters 172, L59.

10. Humphreys, R. M., Strecker, D. W., and Ney, E. P.: 1972, Astrophys. J. 172, 75.

11. Hyland, A. R., Becklin, E. E., Neugebauer, G., and Wallerstein, G.: 1969, Astrophys. J. 158, 619.

12. Johnson, H. L.: 1966, Ann. Rev. Astron. Astrophys. 4, 193.

13. Johnson, H. L., Mitchell, R. I., Iriarte, B., and Wisniewski, W. Z.: 1966, Comm. Lunar Planet. Rec. Lab. 4, 99.

14. Lee, T.: (private communication).

15. Low, F. J.: 1970, Air Force Cambridge Research Laboratories Semi-Annual Technical Report AFCRL-70-0179.

16. Mayall, M. W.: private communication.

17. Neugebauer, G. and Leighton, R. B.: 1969, Two Micron Survey, NASA P-3047.

18. Preston, G. W., Krzeminsky, W., Smak, J., and Williams, J. A.: 1963, Astrophys. J. 137, 401.

19. Geisel, S. L.: 1970, Astrophys. J. 161, L105.

a plot of color excess vs distance for stars in the U.S. Naval Observatory Photoelectric Catalogue (Blanco et al., 1968) that the distance to $\varepsilon$ Aur is approximately $500 \mathrm{pc}$, while spectral classification yields the 1000 pc estimate. 
The nova RS Oph shows strong absorption at $\lambda 6613,5780$, and $5796 \AA$ (Wallerstein, 1958). We find these bands in the nearby cepheid, Y Oph, which suffers about the same amount of interstellar extinction as RS Oph (Kraft, 1961; Sandage and Tammann, 1971) and is thought to be at roughly the same distance. Therefore in this case also we believe that circumstellar diffuse bands are absent. A detailed comparison of the two stars would be very difficult because of blends with atomic lines in Y Oph and uncertainty in the distance to RS Oph. Photometry of RS Oph shows no infrared excess in the $V-K$ color assuming a $K 0$ star to be present (Wallerstein, 1969) that is reddened by 0.85 in $B-V$ (Svolopolous, 1966) but a considerable excess in the $K-N$ color (Geisel et al., 1970). Observations out to $3.5 \mu$ by Harvey (1972) in August and September 1970 confirm the absence of an excess in the near infra-red.

We wish to compare the diffuse band strength or upper limit on it for circumstellar material with the strength to be expected had the reddening material been of the same nature as interstellar dust. The absorption listed in Table I for each star is only a lower limit of the total visual extinction because the scattering term has been omitted. If the ratio of scattering to absorption is similar to that for interstellar material the absorptions must be increased by a factor of three to find the total visual extinction. The proposed factor of three is an upper limit on the range of possible corrections since the particles may be close to the star and have a phase function that favors forward scattering. In the limiting case the scattering will not add to the absorption, since as much light will be scattered into the beam as out of it.

By inspection of Table I we see that a number of stars have sufficient circumstellar absorption that we would expect to see $\lambda 4430$ if it followed the interstellar relationship between $\lambda 4430$ and extinction. If we recognize that the absorption of Table I may be only $\frac{1}{3}$ of the total extinction many of the stars should show $\lambda 4430$, but they.do not.

Gahm and Hultquist (1972) report an anomalous feature at $\lambda 6613$ in the spectra of several late type supergiants, and suggest that it may be the $\lambda 6613$ diffuse band. In our stars we have used a spectra comparator to compare the lines from different stars, and we conclude that the line we see at $6613 \AA$ is the same in each star, rather than being more broad and diffuse for later type stars. We have no stars in common with Gahm and Hultquist.

\section{Discussion}

The lack of circumstellar diffuse bands in stars which have IR excesses may be due to some intrinsic difference between the grains in circumstellar envelopes and those in the general interstellar medium, or to the physical conditions in the near environs of stars. There is some evidence for the reduction of $\lambda 4430$ strength in regions of strong heating (Greenberg, 1968; Stoeckly and Dressler, 1963). On the other hand, A'Hearn (1971) has found evidence of $\lambda 4430$ absorption in reflection nebulae, near hot stars. Although the temperatures in circumstellar dust envelopes are much lower than those in $\mathrm{H}$ II regions they are much higher than in dense interstellar clouds.

If the lack of circumstellar diffuse band absorption is due to some intrinsic property 
of circumstellar dust grains which is different from that of the interstellar grains, then the question arises what that difference may be. Two possibilities are that the composition of the grains is different, or that the size of the grains is different. The size distribution in the circumstellar environment may differ from the interstellar environment because stellar radiation pressure may selectively deplete its circumstellar cloud of the smaller grains. Perhaps our results indicate that circumstellar envelopes around evolved stars are not the main source of the general interstellar dust.

\section{Acknowledgements}

We thank Dr C. R. O'Dell for a valuable discussion of the ratio of absorption to scattering by interstellar grains. This research was supported by NSF grant GP-28882.

\section{References}

A'Hearn, M. F.: 1971, Astron. J. 76, 264.

Blanco, V. M., Demers, S., Douglas, G. G., and Fitzgerald, M. P.: 1968, Publ. U.S. Naval Obs. XXI. Duke, D.: 1951, Astrophys. J. 113, 100.

Gahm, G. F. and Hultquist, L.: 1972, Astron. Astrophys. 16, 329.

Geisel, S. L., Kleinmann, D. E., and Low, F. J.: 1970, Astrophys. J. Letters 161, L101.

Greenberg, J. M.: 1968, in B. M. Middlehurst and L. H. Aller (eds.), Nebulae and Interstellar Matter, University of Chicago Press, p. 221.

Harvey, P.: 1972, private communication.

Herbig, G. H.: 1960, Astrophys. J. Suppl. 4, 337.

Hyland, A. R., Becklin, E. E., Neugebauer, G., and Wallerstein, G.: 1969, Astrophys. J. $158,619$. Kraft, R. P.: 1961, Astrophys. J. 134, 616.

Lockwood, G. W.: preprint.

Lockwood, G. W. and Wing, R. F.: 1971, Astrophys. J. 169, 63.

Sandage, A. and Tamman, G. A.: 1971, Astrophys. J. 167, 293.

Stoeckly, R. and Dressler, K.: 1963, Astrophys. J. 139, 240.

Strom, S. E., Strom, K. M., Brooke, A. L., Bregman, J., and Yost, T.: 1972, Astrophys. J. $171,267$. Svolopolous, S. N.: 1966, Publ. Astron. Soc. Pacific 78, 157.

Van de Hulst, H. C. and De Jong, T.: 1966, Physics 41, 151.

Wallerstein, G.: 1958, Publ. Astron. Soc. Pacific 70, 537.

Wallerstein, G.: 1969, Publ. Astron. Soc. Pacific 81, 672.

Wilson, R.'E.: 1971, Astrophys. J. 170, 529. 